\title{
Gleason Pattern 5 is a Possible Pathologic Predictor for Biochemical Recurrence after Laparoscopic Radical Prostatectomy
}

\author{
Masaomi Ikeda*, Noriyuki Amano, Yusuke Sakata, Tomotsugu Honda, Takashi \\ Tachibana, Shuhei Hirano, Hideyuki Yamashita, Junichiro Ishii, Akira Irie
}

\begin{abstract}
Objective: Several prognostic factors for biochemical recurrence after radical prostatectomy have been reported, including initial prostate-specific antigen level, Gleason score, positive surgical margin, and seminal vesicle invasion. Here we investigate whether Gleason pattern 5 is a predictor for biochemical recurrence. Methods: This retrospective study included 168 patients who underwent laparoscopic radical prostatectomy from 2006 to 2015. The relationship between biochemical recurrence after laparoscopic radical prostatectomy and the presence of Gleason pattern 5, even as a tertiary pattern, was investigated. Biochemical recurrence was defined when the prostate-specific antigen level rose to $>0.2 \mathrm{ng} / \mathrm{ml}$ after having decreased to $<0.1 \mathrm{ng} / \mathrm{ml}$ following laparoscopic radical prostatectomy. Biochemical recurrence-free survival was estimated by the Kaplan-Meier method. Multivariate analysis was performed using a Cox proportional hazards regression model. Results: The median age was 66 years, median initial prostate-specific antigen level was $6.9 \mathrm{ng} / \mathrm{ml}$, and median follow-up period was 47.3 months. Biochemical recurrence was recognized in 27 patients (16.1\%) after laparoscopic radical prostatectomy, and 5-year biochemical recurrence-free survival was $78.6 \%$. Gleason pattern 5 was noted in 5 patients as the primary pattern, in 10 as the secondary pattern, and in 5 as the tertiary pattern. According to multivariate analysis, presence of Gleason pattern $5(\mathrm{HR}=4.75, \mathrm{p}=0.001)$ and positive surgical margin $(\mathrm{HR}=4.66, \mathrm{p}=0.001)$ were independent predictive factors for biochemical recurrence-free survival. Conclusion: Gleason pattern 5 appears to be an important predictive factor for biochemical recurrence after laparoscopic radical prostatectomy.
\end{abstract}

Keywords: Gleason pattern 5- biochemical recurrence- prostate cancer- laparoscopic radical prostatectomy

Asian Pac J Cancer Prev, 20 (3), 783-788

\section{Introduction}

Radical prostatectomy is a common treatment option for localized and locally advanced prostate cancer. In the late 1990s, laparoscopic radical prostatectomy (LRP) became an accepted alternative to open radical prostatectomy for the management of localized prostate cancer (Goeman et al., 2006; Guillonneau et al., 2003). Studies have shown that there is no differences between LRP and open radical prostatectomy with regard to oncological outcomes such as biochemical recurrence-free survival (BCRFS) rate and positive surgical margin (SM) rate (Ficarra et al., 2009; Drouin et al., 2009). Biochemical recurrence (BCR) occurs in an approximately $30 \%$ of patients after radical prostatectomy for localized prostate cancer (Ficarra et al., 2009; Roehl et al., 2004; Hull et al., 2002).

Many studies have reported predictive factors for BCR after open radical prostatectomy such as initial prostate-specific antigen (PSA) level, prostate volume, positive SM, pathological T stage, and the Gleason score (Uhlman et al., 2010; Boorjian et al., 2010; Pfitzenmaier et al., 2008; Chalieopanyarwong et al., 2017). The Gleason score, especially Gleason pattern 5 (GP5), is an important predictive factor for $\mathrm{BCR}$, clinical recurrence, and cancer-specific survival (Sabolch et al., 2011; Loeb et al., 2011; Nanda et al., 2009; Cheng et al., 2007). In this study, we investigated the relationship between BCR after LRP and the presence of GP5, including as a tertiary pattern.

\section{Materials and Methods}

\section{Patient selection}

This study was a retrospective analysis of 180 patients who underwent LRP for clinically localized and locally advanced prostate cancer from 2006 to 2015 at the Kitasato Institute Hospital. Among them, 12 patients who received perioperative hormonal therapy were

Department of Urology, Kitasato University Kitasato Institute Hospital, Tokyo, Japan. *For Correspondence: ikeda.masaomi@grape.plala.or.jp 
excluded from the study. The remaining 168 patients were treated with LRP by a single surgeon, although the procedure changed periodically. Briefly, from 2006 to 2008 the approach for the excision was conducted intraperitoneally, but from 2009 to 2015 the retroperitoneal approach was used. Obturator lymph node dissection was conducted only in cases with an intermediate or high risk according to D'Amico risk classification. A nerve-sparing procedure (bilateral or lateral) was also performed only in selected cases considering the clinical background for maintaining cancer control. This study was approved by the institutional review board and conducted in accordance with the Declaration of Helsinki.

\section{Histopathological examination}

For histopathological examination, LRP specimens were fixed and the distal and proximal parts of the prostate were amputated (True, 1994). The diagnosis of adenocarcinoma was based on hematoxylin/eosin-stained slides. Histological classification was done according to the International Society of Urological Pathology Consensus on Gleason grading of prostate cancer (Epstein et al., 2005). Primary and secondary Gleason scores were recorded according to the prevalence of the most prominent grade in the largest tumor. Tertiary Gleason pattern was defined as the third most frequent pattern identified in the specimen regardless of amount and anatomical location.

\section{Biochemical recurrence}

All patients were followed up by routine serum PSA assay every 3 months for the first 2 years, and every 6 months thereafter. BCR was defined when two consecutive PSA levels were higher than $0.2 \mathrm{ng} / \mathrm{ml}$. A patient whose PSA level had never been lower than $0.1 \mathrm{ng} / \mathrm{ml}$ following LRP was also considered to have BCR. Computed tomography, magnetic resonance imaging, and bone scans were performed when BCR or clinical disease progression was suspected. BCRFS time was calculated from LRP to the date of documented BCR.

\section{Statistical analysis}

Clinical and pathological variables were compared among groups using the chi-square test, Fisher's exact test, and Kruskal-Wallis test. BCRFS curves were estimated by the Kaplan-Meier method and compared among groups with a log rank test. Univariate and multivariate Cox proportional hazards regression models addressed the associations of BCRFS with age at surgery, initial PSA $(<10 \mathrm{ng} / \mathrm{ml}$ vs. $\geq 10 \mathrm{ng} / \mathrm{ml}$ ), pathological T stage (pT2 vs. $\geq$ pT3), GP5 (absent vs. present), SM (negative vs. positive), extraprostatic extension (negative vs. positive), seminal vesicle invasion (negative vs. positive), and lymphovascular invasion (absent vs. present). All analyses were performed with Stata ver. 13 for Windows (Stata, Chicago, IL, USA). A p $<0.05$ was chosen to indicate statistical significance, and all $\mathrm{p}$ values were two-sided.

\section{Results}

Table 1 shows the clinical and pathological characteristics of all patients divided by the presence or absence of GP5. The median age at time of surgery was 66 years (IQR $=61-69$ years), median initial PSA level was $6.9 \mathrm{ng} / \mathrm{ml}(\mathrm{IQR}=5.1-9.9 \mathrm{ng} / \mathrm{ml})$, and median follow-up period was 47.3 months $(\mathrm{IQR}=30.0-71.2$ months). Bilateral obturator lymph node dissection was performed in 54 patients (32\%). The median number of removed lymph nodes was $4(\mathrm{IQR}=2-6)$, but no positive lymph node was observed in any patient. Although there were statistically significant differences in biopsy Gleason score, D'Amico risk classification, pathological Gleason score, lymphovascular invasion, seminal vesicle invasion, and BCR between the GP5 presence and absence groups, there was no statistically significant difference regarding SM. GP5 was recognized in 20 patients (12\%), and these were confirmed as primary in 5 , as secondary in 10 , and as tertiary in 5. The dominant Gleason score of all patients with tertiary GP5 was $4+3$.

BCR was recognized in 27 patients (16\%) after LRP, with GP5 present in 9 patients and absent in 18. Table 2 shows the details of clinicopathological characteristics of the 27 patients with BCR. Among them, 13 patients
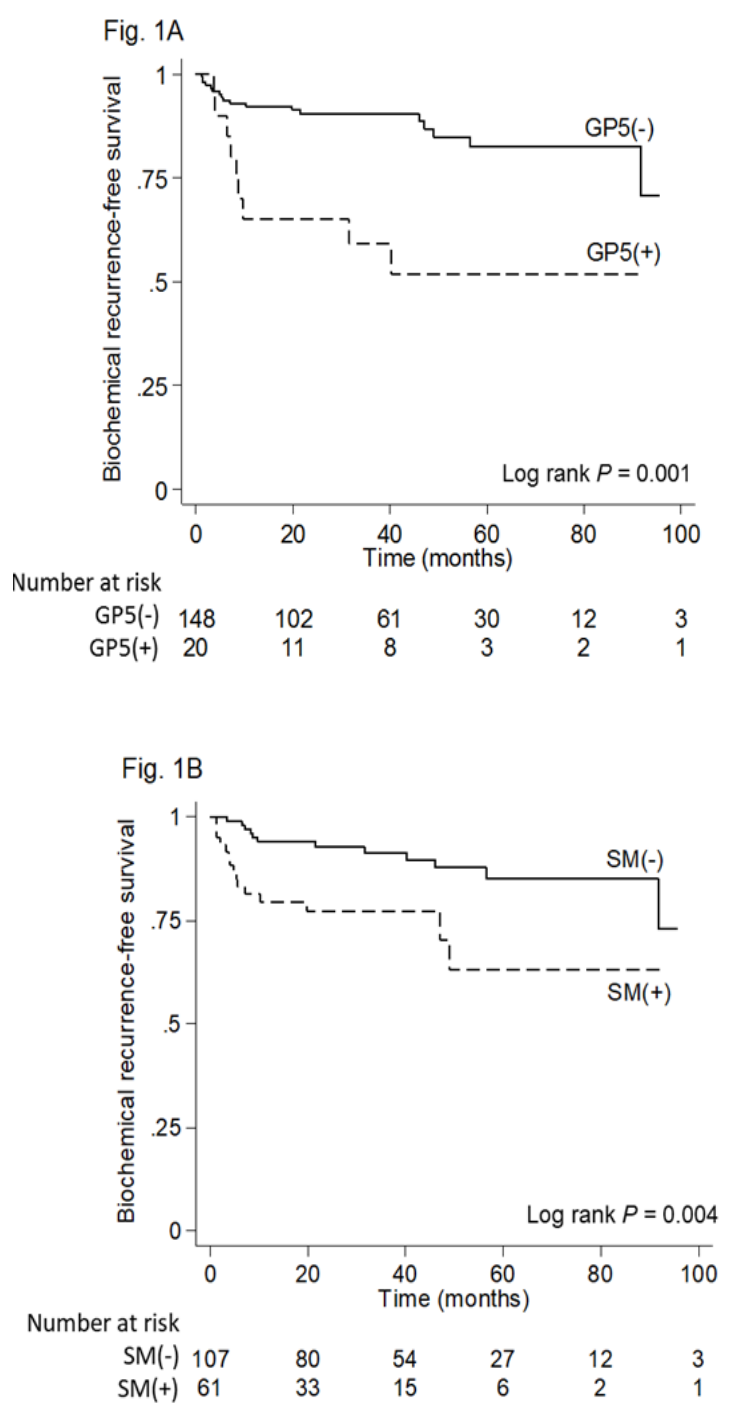

Figure 1. Kaplan-Meier Curves of Biochemical Recurrence-Free Survival Stratified by Gleason Pattern 5 (A) and Surgical Margin (B). 
Table 1. Clinical and Pathological Characteristics of All 168 Patients Classified by Gleason Pattern 5 (GP5)

\begin{tabular}{|c|c|c|c|c|}
\hline Variable & $\begin{array}{l}\text { All patients } \\
(\mathrm{n}=168)\end{array}$ & $\begin{array}{l}\text { GP5 absent } \\
(\mathrm{n}=148)\end{array}$ & $\begin{array}{l}\text { GP5 present } \\
(\mathrm{n}=20)\end{array}$ & $\mathrm{p}$ value \\
\hline Age of surgery, years, median (IQR) & $66(61-69)$ & $66(61-69)$ & $67(61-69)$ & 0.654 \\
\hline Initial prostate-specific antigen, $\mathrm{ng} / \mathrm{ml}$, median (IQR) & $6.9(5.1-9.9)$ & $6.9(5.0-9.8)$ & $7.2(5.4-10.8)$ & 0.425 \\
\hline Biopsy Gleason score (\%) & & & & 0.001 \\
\hline$\leq 6$ & $48(29 \%)$ & $47(98 \%)$ & $1(2 \%)$ & \\
\hline 7 & $88(52 \%)$ & $80(91 \%)$ & $8(9 \%)$ & \\
\hline $8-10$ & $32(19 \%)$ & $21(66 \%)$ & $11(34 \%)$ & \\
\hline Clinical T stage $(\%)$ & & & & 0.522 \\
\hline $\mathrm{cT} 1 \mathrm{c}$ & $143(85 \%)$ & $127(89 \%)$ & $16(11 \%)$ & \\
\hline cT2 & $22(13 \%)$ & $18(82 \%)$ & $4(18 \%)$ & \\
\hline cT3 & $3(2 \%)$ & $3(100 \%)$ & $0(0 \%)$ & \\
\hline D'Amico risk classification (\%) & & & & 0.001 \\
\hline Low & $41(24 \%)$ & $41(100 \%)$ & $0(0 \%)$ & \\
\hline Intermediate & $87(52 \%)$ & $78(90 \%)$ & $9(10 \%)$ & \\
\hline High & $40(24 \%)$ & $29(73 \%)$ & $11(27 \%)$ & \\
\hline Pathological T stage (\%) & & & & 0.066 \\
\hline pT2 & $138(82 \%)$ & $124(90 \%)$ & $14(10 \%)$ & \\
\hline pT3a & $21(13 \%)$ & $18(86 \%)$ & $3(14 \%)$ & \\
\hline pT3b & $7(4 \%)$ & $4(57 \%)$ & $3(43 \%)$ & \\
\hline pT4 & $2(1 \%)$ & $2(100 \%)$ & $0(0 \%)$ & \\
\hline Pathological Gleason score (\%) & & & & 0.001 \\
\hline$\leq 6$ & $36(21 \%)$ & $36(100 \%)$ & $0(0 \%)$ & \\
\hline 7 & $111(66 \%)$ & $106(95 \%)$ & $5(5 \%)$ & \\
\hline $8-10$ & $21(13 \%)$ & $6(29 \%)$ & $15(71 \%)$ & \\
\hline Lymphovascular invasion (\%) & & & & 0.02 \\
\hline Absent & $153(91 \%)$ & $138(90 \%)$ & $15(10 \%)$ & \\
\hline Present & $15(9 \%)$ & $10(67 \%)$ & $5(33 \%)$ & \\
\hline Surgical margin $(\%)$ & & & & 0.626 \\
\hline Negative & $107(64 \%)$ & $93(87 \%)$ & $14(13 \%)$ & \\
\hline Positive & $61(36 \%)$ & $55(90 \%)$ & $6(10 \%)$ & \\
\hline Extraprostatic extension (\%) & & & & 0.326 \\
\hline Negative & $141(84 \%)$ & $126(89 \%)$ & $15(11 \%)$ & \\
\hline Positive & $27(16 \%)$ & $22(81 \%)$ & $5(19 \%)$ & \\
\hline Seminal vesicle invasion $(\%)$ & & & & 0.037 \\
\hline Negative & $161(96 \%)$ & $144(89 \%)$ & $17(11 \%)$ & \\
\hline Positive & $7(4 \%)$ & $4(57 \%)$ & $3(43 \%)$ & \\
\hline Lymph node status (\%) & & & & 0.068 \\
\hline $\mathrm{pN} 0$ & $54(32 \%)$ & $44(81 \%)$ & $10(19 \%)$ & \\
\hline $\mathrm{pNx}$ & $114(68 \%)$ & $104(91 \%)$ & $10(9 \%)$ & \\
\hline $\mathrm{pN} 1$ & $0(0 \%)$ & $0(0 \%)$ & $0(0 \%)$ & \\
\hline Biochemical recurrence $(\%)$ & & & & 0.001 \\
\hline No & $141(84 \%)$ & $130(92 \%)$ & $11(8 \%)$ & \\
\hline Yes & $27(16 \%)$ & $18(67 \%)$ & $9(33 \%)$ & \\
\hline Follow-up, months, median (IQR) & $47.3(30.0-71.2)$ & $44.0(29.5-69.8)$ & $60.8(46.6-74.8)$ & 0.091 \\
\hline
\end{tabular}

(48\%) received salvage radiotherapy, $9(33 \%)$ received androgen-deprivation therapy, $3(11 \%)$ received both radiotherapy and androgen-deprivation therapy, and 2 $(8 \%)$ did not receive any treatment. There was no clinical recurrence nor metastasis during the observation period.
There was no cancer death in the follow-up period, but 3 patients died of other causes.

The BCRFS rate at 5 years was $78.6 \%$ in all patients. Table 3 summarizes the univariate and multivariate analyses for BCRFS. According to multivariate Cox 
Table 2. Clinicopathological Characteristics of 27 Patients with Biochemical Recurrence

\begin{tabular}{lcc}
\hline Variable & & $\mathrm{n}=27$ \\
\hline Age of surgery, years & median (IQR) & $66(62-69)$ \\
Initial prostate-specific antigen, ng/ml & median (IQR) & $7.8(6.1-11.0)$ \\
D'Amico risk & low/intermediate/high & $6 / 14$ \\
Pathological T stage & pT2/pT3/pT4 & $21 / 4 / 2$ \\
Pathological Gleason score & $\leq 6 / 7 / 8-10$ & $5 / 18$ \\
Gleason pattern 5 & present/absent & $9 / 18$ \\
Nerve spare & yes/no & $14 / 13$ \\
Surgical margin & positive/negative & $15 / 12$ \\
Lymphovascular invasion & present/absent & $21 / 6$ \\
Extraprostatic extension & positive/negative & $3 / 24$ \\
\hline
\end{tabular}

proportional hazards regression analysis, presence of GP5 $(\mathrm{HR}=4.75, \mathrm{p}=0.001)$ and positive $\mathrm{SM}(\mathrm{HR}=4.66, \mathrm{p}=$ $0.001)$ were independent predictive factors for BCRFS. Kaplan-Meier curves of BCRFS according to the presence of GP5 are shown in Figure 1A. The BCRFS rate at 5 years was $82.9 \%$ versus $50.8 \%$ in the GP5 absent and present groups, respectively $(p=0.001)$. Kaplan-Meier curves of BCRFS according to SM status are shown in Figure 1B. BCRFS rate at 5 years was $85.6 \%$ versus $63.7 \%$ in

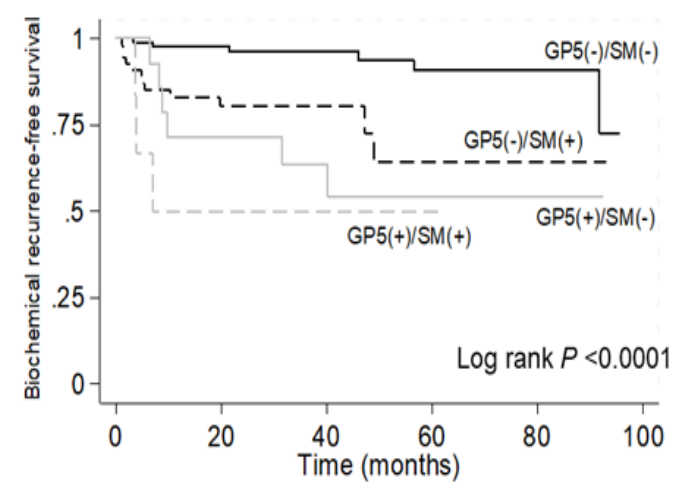

Number at risk

$\begin{array}{ccccccc}\text { GP5(-) / SM(-) } & 93 & 71 & 47 & 25 & 10 & 2 \\ \text { GP5(-) / SM(+) } & 55 & 31 & 14 & 5 & 2 & 1 \\ \text { GP5(+) / SM(-) } & 14 & 9 & 7 & 2 & 2 & 1 \\ \text { GP5(+) / SM(+) } & 6 & 2 & 1 & 1 & 0 & 0\end{array}$

Figure 2. Four Groups of Kapan-Meier Curves of Biochemical Recurrence-Free Survival Stratified by Gleason Pattern 5 and Surgical Margin the negative and positive SM groups, respectively $(\mathrm{p}=$ 0.004). Kaplan-Meier curves of BCRFS according to the GP5 and SM status are shown in Figure 2. The 5-year BCRFS rate was $91.3 \%, 64.8 \%, 53.6 \%$, and $50.0 \%$ in the absent GP5/negative SM, absent GP5/positive SM, present GP5/negative SM, and present GP5/positive SM, respectively $(\mathrm{p}<0.0001)$.

\section{Discussion}

Many prognostic factors, such as initial PSA, positive SM, pathological T stage, and Gleason score, that predict BCR after radical prostatectomy. Gleason score represents a highly important prognostic parameter of a radical prostatectomy specimen. In particular, GP5, even as a tertiary pattern, is thought to be a significant predictor. The Gleason system accounts only for the two most dominant patterns, however, and prostate cancer is a heterogeneous disease often with the presence of different tumor foci and variable grades of aggressiveness within the prostate gland. In 2005, the role of the Gleason score as a prognostic factor was largely revised. The International Society of Urological Pathology decided that the Gleason score should include the primary and secondary pattern, with a separate comment on the presence of a tertiary pattern of higher grade in the radical prostatectomy specimen.

The present study demonstrated that clinical outcomes of patients with GP5 and positive SM after LRP were worse than those without GP5 and negative SM.

Table 3. Univariate and Multivariate Cox Proportional Hazards Analysis for the Prediction of Biochemical RecurrenceFree Survival

\begin{tabular}{lccc}
\hline Parameter & Univariate & Multivariate \\
& $\mathrm{p}$ & HR $(95 \% \mathrm{CI})$ & $\mathrm{p}$ \\
\hline Age $\geq 66$ years (reference: $<66$ years) & 0.500 & & \\
Initial prostate-specific antigen $\geq 10 \mathrm{ng} / \mathrm{ml}$ (reference: $<10 \mathrm{ng} / \mathrm{ml}$ ) & 0.203 & & \\
$\geq \mathrm{pT} 3$ (reference: $\mathrm{pT}$ ) & 0.369 & & 0.001 \\
Present Gleason pattern 5 (reference: absent) & 0.001 & $4.75(1.97-11.4)$ & 0.001 \\
Positive surgical margin (reference: negative) & 0.006 & $4.66(1.91-11.4)$ & \\
Positive extraprostatic extension (reference: negative) & 0.598 & & \\
Positive seminal vesicle invasion (reference: negative) & 0.096 & & \\
Present lymphovascular invasion (reference: absent) & 0.177 & & \\
\hline
\end{tabular}


In addition, presence of GP5 and positive SM were independent predictive factors of BCRFS in our univariate and multivariate analyses.

Lim et al., (2013) reported differences in oncological outcome between the primary GP5 and the secondary GP5 in Gleason score 9 patients who underwent roboticassisted radical prostatectomy. The primary GP5 patients were associated with a significantly greater proportion of lymph node involvement and had a significantly poorer BCRFS compared with the secondary GP5 patients. In our study, 3-year BCRFS in the secondary GP5 patients reflected a better prognosis compared with the primary GP5 patients ( $89.5 \%$ vs. $60.0 \%$; data not shown). In addition, several studies reported that the presence of tertiary GP5 is also a significant and independent factor of BCR after radical prostatectomy in cases with Gleason score 7 (Lucca et al., 2015; Adam et al., 2014; Sim et al., 2008). Sim et al., (2008) investigated 509 patients with Gleason score 7 among 1110 cases of clinical localized prostate cancer who underwent radical prostatectomy. Tertiary GP5 was present in 66 of these 509 cases (13\%). Patients with Gleason score 7 without tertiary GP5 had higher PSA recurrence-free probability than those with tertiary GP5. In addition, patients with Gleason sum $4+3+5$ had a lower PSA recurrence-free probability than Gleason sum 3+4, Gleason sum 3+4+5, and Gleason sum $4+3$. Multivariate analysis showed that tertiary GP5 was associated with a higher rate of $\mathrm{BCR}(\mathrm{HR}=1.78,95 \% \mathrm{CI}$ $=1.00-3.17)$. Sauter et al., (2017) recently examined how the occupancy rate of tertiary GP5 affects BCR in a cohort of 13261 patients who underwent radical prostatectomy using a new integrated quantitative Gleason score. As the integrated quantitative Gleason score increased, that is, the greater the occupancy rate of tertiary GP5, the higher the $\mathrm{BCR}$ rate.

Positive SMs in radical prostatectomy specimens also have been consistently associated with an increased risk of BCR. Several studies reported that the incidence of positive $\mathrm{SM}$ is equivalent among open radical prostatectomy, LRP, and robot-assisted radical prostatectomy (Tewari et al., 2012; Touijer et al., 2008). Novara et al., (2012) reported the average rate of positive SMs in a contemporary robot-assisted radical prostatectomy series was $15 \%$ (range: 6.5-32\%). However, the stage-specific rates were $9 \%$ for $\mathrm{pT} 2,37 \%$ for $\mathrm{pT} 3$, and $50 \%$ for $\mathrm{pT} 4$, supporting the notion that the more extensive the cancer, the higher the risk of positive SM. On the other hand, Yossepowich et al., (2014) reviewed the relationship between positive SM after radical prostatectomy and oncological outcomes; positive SM was associated with a 1.6- to 5-fold increased hazard of BCR, but its association with cancer-specific survival was unclear. Chalfin et al., (2012) investigated 4,569 men who underwent radical prostatectomy at a median follow-up of 10 years. In multivariate analysis, positive $\mathrm{SM}(\mathrm{HR}=1.4, \mathrm{p}=0.036)$ showed a significantly adverse effect on cancer-specific survival, but radical prostatectomy Gleason score $(\mathrm{HR}=5.7-12.6, \mathrm{p}<0.001)$ and pathological stage $(\mathrm{HR}=2.2-11.0, \mathrm{p}<0.001)$ were stronger predictors. Other studies did not find positive $\mathrm{SM}$ to be an independent predictor of cancer-specific survival (Boorjian et al., 2010; Pfitzenmaier et al., 2008;
Mauermann et al., 2013). Therefore, adjuvant radiotherapy is recommended as it may affect BCR favorably, but whether the adjuvant treatment also affects cancer-specific survival remains debatable and further study is required.

In our study, 25 patients received salvage radiotherapy or androgen-deprivation therapy for BCR after LRP, and there was no clinical or metastatic recurrence after salvage treatment in the follow-up period. However, several studies reported that the presence of GP5 was the strongest pathological predictor of outcomes of salvage treatment after radical prostatectomy. Taguchi et al., (2016) investigated 116 patients with pT2-3N0M0 prostate cancer who received salvage treatment for BCR after radical prostatectomy. Multivariate analysis demonstrated that GP5 was the only independent predictor of poor outcome $(\mathrm{HR}=3.48, \mathrm{p}=0.022)$. Jackson et al., (2013) investigated 575 patients who underwent primary radical prostatectomy and subsequently received salvage radiotherapy. A total of $60(10.7 \%)$ patients had primary, secondary, or tertiary GP5. On multivariate analysis, GP5 was the most adverse pathologic predictor of $\mathrm{BCR}(\mathrm{HR}=2.9, \mathrm{p}<0.0001)$, distant metastasis $(\mathrm{HR}=14.8, \mathrm{p}<0.0001)$, and cancerspecific survival $(\mathrm{HR}=5.7, \mathrm{p}<0.0001)$. Although we could not investigate the oncological outcome after salvage treatment because clinical recurrence or metastasis was not recognized in any patients in the present study, the presence of GP5 including tertiary GP5 was thought to be an important pathological feature of BCR.

This study had several limitations, including the limited number of cases, single center cohort, and relatively short observation period (4 years) after LRP. Further studies with a larger population and longer follow-up might be required to confirm the results.

The presence of GP5 and positive SM appear to be an important pathological predictors for BCR after LRP. These risk factors may assist in clinical decision-making regarding adjuvant treatment and follow-up schedule.

\section{Statement conflict of Interest}

This research did not receive any specific grants from funding agencies in the public, commercial, or not-for-profit sectors.

\section{Acknowledgements}

There are no especial acknowledgements.

\section{References}

Adam M, Hannah A, Budaus L, et al (2014). A tertiary Gleason pattern in the prostatectomy specimen and its association with adverse outcome after radical prostatectomy. J Urol, 192, 97-101.

Boorjian SA, Karnes RJ, Crispen PL, et al (2010). The impact of positive surgical margins on mortality following radical prostatectomy during the prostate specific antigen era. $J$ Urol, 183, 1003-9.

Chalfin HJ, Dinizo M, Trock BJ, et al (2012). Impact of surgical margin status on prostate-cancer-specific mortality. BJU Int, 110, 1684-9.

Chalieopanyarwong V, Attawettayanon W, Kanchanawanichkul W, et al (2017). The prognostic factors of biochemical

Asian Pacific Journal of Cancer Prevention, Vol 20 
recurrence-free survival following radical prostatectomy. Asian Pac J Cancer Prev, 18, 2555-9.

Cheng L, Davidson DD, Lin H, Koch MO (2007). Percentage of Gleason pattern 4 and 5 predicts survival after radical prostatectomy. Cancer, 110, 1967-72.

Drouin SJ, Vaessen C, Hupertan V, et al (2009). Comparison of mid-term carcinologic control obtained after open, laparoscopic, and robot-assisted radical prostatectomy for localized prostate cancer. World J Urol, 27, 599-605.

Epstein JI, Allsbrook WC Jr., Amin MB, Egevad LL, Isup Grading Committee (2005). The 2005 International Society of Urological Pathology (ISUP) Consensus Conference on Gleason Grading of Prostatic Carcinoma. Am J Surg Pathol, 29, 1228-42.

Ficarra V, Novara G, Artibani W, et al (2009). Retropubic, laparoscopic, and robot-assisted radical prostatectomy: a systematic review and cumulative analysis of comparative studies. Eur Urol, 55, 1037-63.

Goeman L, Salomon L, La De Taille A, et al (2006). Long-term functional and oncological results after retroperitoneal laparoscopic prostatectomy according to a prospective evaluation of 550 patients. World J Urol, 24, 281-8.

Guillonneau B, el-Fettouh H, Baumert H, et al (2003). Laparoscopic radical prostatectomy: oncological evaluation after 1,000 cases a Montsouris Institute. J Urol, 169, 1261-6.

Hull GW, Rabbani F, Abbas F, et al (2002). Cancer control with radical prostatectomy alone in 1,000 consecutive patients. J Urol, 167, 528-34.

Jackson W, Hamstra DA, Johnson S, et al (2013). Gleason pattern 5 is the strongest pathologic predictor of recurrence, metastasis, and prostate cancer-specific death in patients receiving salvage radiation therapy following radical prostatectomy. Cancer, 119, 3287-94.

Lim SK, Kim KH, Shin TY, et al (2013). Gleason $5+4$ has worse oncological and pathological outcomes compared with Gleason 4+5: significance of Gleason 5 pattern. Ann Surg Oncol, 20, 3127-32.

Loeb S, Schaeffer EM, Trock BJ, et al (2010). What are the outcomes of radical prostatectomy for high-risk prostate cancer?. Urology, 76, 710-4.

Lucca I, Shariat SF, Briganti A, et al (2015). Validation of tertiary Gleason pattern 5 in Gleason score 7 prostate cancer as an independent predictor of biochemical recurrence and development of a prognostic model. Urol Oncol, 33, 71.e21-6.

Mauermann J, Fradet V, Lacombe L, et al (2013). The impact of solitary and multiple positive surgical margins on hard clinical end points in 1712 adjuvant treatment-naive pT2-4 N0 radical prostatectomy patients. Eur Urol, 64, 19-25.

Nanda A, Chen MH, Renshaw AA, D’Amico AV (2009). Gleason pattern 5 prostate cancer: further stratification of patients with high-risk disease and implications for future randomized trials. Int J Radiat Oncol Biol Phys, 74, 1419-23.

Novara G, Ficarra V, Mocellin S, et al (2012). Systematic review and meta-analysis of studies reporting oncologic outcome after robot-assisted radical prostatectomy. Eur Urol, 62, 382-404.

Pfitzenmaier J, Pahernik S, Tremmel T, et al (2008). Positive surgical margins after radical prostatectomy: Do they have an impact on biochemical or clinical progression?. BJU Int, 102, 1413-8.

Roehl KA, Han M, Ramos CG, Antenor JA, Catalona WJ (2004). Cancer progression and survival rates following anatomical radical retropubic prostatectomy in 3,478 consecutive patients: long-term results. J Urol, 172, 910-4.

Sabolch A, Feng FY, Daignault-Newton S, et al (2011). Gleason pattern 5 is the greatest risk factor for clinical failure and death from prostate cancer after dose-escalated radiation therapy and hormonal ablation. Int J Radiat Oncol Biol Phys, 81, e351-60.

Sauter G, Clauditz T, Steurer S, et al (2018). Integrating tertiary Gleason 5 patterns into quantitative Gleason grading in prostate biopsies and prostatectomy specimens. Eur Urol, 73, 674-83.

Sim HG, Telesca D, Culp SH, et al (2008). Tertiary Gleason pattern 5 in Gleason 7 prostate cancer predicts pathological stage and biochemical recurrence. J Urol, 179, 1775-9.

Taguchi S, Shiraishi K, Fukuhara H, et al (2016). Impact of Gleason pattern 5 including tertiary pattern 5 on outcomes of salvage treatment for biochemical recurrence in pT2-3N0M0 prostate cancer. Int J Clin Oncol, 21, 975-80.

Tewari A, Sooriakumaran P, Bloch DA, et al (2012). Positive surgical margin and perioperative complication rates of primary surgical treatments for prostate cancer: a systematic review and meta-analysis comparing retropubic, laparoscopic, and robotic prostatectomy. Eur Urol, 62, 1-15.

Touijer K, Eastham JA, Secin FP, et al (2008). Comprehensive prospective comparative analysis of outcomes between open and laparoscopic radical prostatectomy conducted in 2003 to 2005. J Urol, 179, 1811-7.

True LD (1994). Surgical pathology examination of the prostate gland. Practice survey by American Society of Clinical Pathologists. Am J Clin Pathol, 102, 572-9.

Uhlman MA, Sun L, Stackhouse DA, et al (2010). Tumor volume, tumor percentage involvement, or prostate volume: Which is predictive of prostate-specific antigen recurrence?. Urology, 75, 460-6.

Yossepowitch O, Briganti A, Eastham JA, et al (2014). Positive surgical margins after radical prostatectomy: a systematic review and contemporary update. Eur Urol, 65, 303-13.

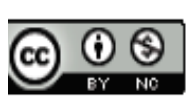

This work is licensed under a Creative Commons AttributionNon Commercial 4.0 International License. 